\title{
Método para la evaluación de la Usabilidad del Software del Voto Electrónico Presencial - UsabVEP
}

\section{Method for the evaluation of the Usability of the Electronic Voting Software - UsabVEP}

\author{
Néstor Núñez Marinovich ${ }^{1}$, Nora La Serna Palomino ${ }^{2}$ \\ Universidad Nacional Mayor de San Marcos, Facultad de Ingeniería de Sistemas e Informática. Lima, Perú \\ ${ }^{1}$ Email: marinovich1983@gmail.com \\ 2Email: nlasernap@unmsm.edu.pe
}

\begin{abstract}
Resumen
La evaluación de la usabilidad del software es importante en el desarrollo de sistemas informáticos porque revela problemas de diseño e interactividad. El software del voto electrónico presencial propone grandes retos de usabilidad debido a que automatiza tareas tales como: comprobación de la identidad del elector, emisión del voto, escrutinio, emisión de reportes de resultados, transmisión y presentación de resultados. Considerando la existencia de métodos de usabilidad que están enfocados a distintos ámbitos específicos o siendo algunos muy generales surge la necesidad de plantear un método adaptado al software de voto electrónico presencial que permita detectar deficiencias en usabilidad. En este trabajo se desarrolló un método para evaluar la usabilidad del sistema del voto electrónico presencial denominado UsabVEP, este se aplicó en el software del proceso electoral de un distrito de Lima, se analizaron los resultados, se validó y se propuso mejoras al sistema. El método plantea dos fases: 1. Evaluación Heurística y 2. Evaluación con usuarios. Los resultados muestran que el método desarrollado encontró un $46 \%$ más de problemas de usabilidad respecto al método de Jakob Nielsen, tomado como referente, para el software del voto electrónico presencial en el Perú.
\end{abstract}

Palabras clave: Evaluación con usuarios; Evaluación heurística; Método de evaluación; Usabilidad; Voto electrónico.

\begin{abstract}
The evaluation of software usability have an important role in the development of computer systems, because it reveals design and interactivity problems. The electronic voting software proposes great usability challenges because it automates task such as: verification of the identity of the voter, voting, scrutiny, results reports, transmission and presentation of results. Considering the existence of usability methods that are focused on different specific areas or being some very general, the need arises to propose a method adapted to the electronic voting software that allows to detect deficiencies in usability. In this work a method was developed to evaluate the usability of the electronic voting system called UsabVEP, this was applied in the electoral process software of a district of Lima, the results were analyzed, the system was validated and improvements were proposed. The method proposes two phases: 1 . Heuristic evaluation and 2. Evaluation with users. The results show that the method developed found $46 \%$ more usability problems compared to the method of Jakob Nielsen, taken as a reference, for the electronic voting software in Peru.
\end{abstract}

Keywords: User evaluation; Heuristic evaluation; Evaluation method; Usability; Electronic voting.

Correspondencia:

Dirección: Universidad Nacional Mayor de San Marcos, Facultad de Ingeniería de Sistemas e Informática. Calle Germán Amézaga № 375 , Ciudad Lima 1.

Recibido 01/03/2019 - Aceptado 10/06/2019

Citar como:

Nuñez, N. \& La Serna, N. (2019) Método para la evaluación de la Usabilidad del Software del Voto Electrónico Presencial - UsabVEP. Revista Peruana de Computación y Sistemas, 2(1):27-36. http://dx.doi.org/10.15381/rpcs.v2i1.16361

(C) Los autores. Este artículo es publicado por la Revista Peruana de Computación y Sistemas de la Facultad de Ingeniería de Sistemas e Informáticade la Universidad Nacional Mayor de San Marcos. Este es un artículo de acceso abierto, distribuido bajo los términos de la licencia Creative Commons Atribucion - No Comercia_Compartir Igual 4.0 Internacional. (http://creativecommons.org/licenses/by-nc-sa/4.0/) que permite el uso no comercial, distribución y reproducción en cualquier medio, siempre que la obra original sea debidamente citada. 


\section{Introducción}

El voto electrónico presencial (VEP) es una forma de votación basada en medios electrónicos que hace uso de componentes hardware y software que permiten automatizar tareas tales como: comprobación de la identidad del elector, emisión del voto, escrutinio, emisión de reportes de resultados, transmisión y presentación de resultado [15]. El software debe cumplir unos requisitos mínimos de usabilidad para el uso de los votantes. Para lograr esto se deben realizar determinadas pruebas al sistema VEP conocidas como evaluaciones de usabilidad. Entendiéndose por usabilidad a la capacidad del software de ser comprendido, aprendido, usado, atractivo y accesible para el usuario en condiciones específicas de uso [10].

Según [19] en las evaluaciones de usabilidad se emplean heurísticas y pruebas con usuarios finales del sistema. Ambos métodos proporcionan una valiosa visión de los problemas de usabilidad, se complementan una con otra y ninguna sustituye a la otra. Se define heurística como guía, principio o regla en los que se basa el evaluador para llevar a cabo la evaluación de usabilidad [12]. La falta o incumplimiento de alguna heurística se estaría ante un problema de usabilidad.

De acuerdo con [5] se deben elaborar heurísticas relacionadas al problema en estudio con el fin de encontrar de manera pertinente los problemas de usabilidad del software. En el presente trabajo se establecen los heurísticos apropiados para software de voto electrónico. Se consideraron algunas heurísticas de uso general, otras se adaptaron de acuerdo a las funcionalidades del sistema, a la organización del proceso, a las facilidades que deben tener los usuarios de diferentes regiones y edades, a la información que debe proporcionar el sistema para la emisión de los resultados, entre otros criterios que se deben tener en cuenta para estos procesos.

El no tener una adecuada usabilidad en un software de votación electrónica, se tendrían problemas tales como: dificultad de encontrar rápidamente elementos en la pantalla, inconvenientes al interactuar con usuarios iletrados o con otros idiomas, instrucciones ilegibles o difíciles de entender, problemas de interactuar con usuarios con limitaciones visuales o motoras, dificultad de aprender a usarlo, insatisfacción del usuario final, etc. [6], [11].

Con la evolución de las tecnologías, los países a nivel mundial utilizan cada vez más sistemas de votación electrónica como un elemento de apoyo importante en la elección de gobiernos locales, regionales y nacionales [21]. De otra manera, la usabilidad mide el grado de satisfacción de un usuario al utilizar un software [6]. Razones que justifican la realización del presente trabajo, que tuvo como objetivo desarrollar un método para la evaluación de la usabilidad del software del Voto Electrónico Presencial que se denominó UsabVEP. Luego, se implementó el método en un proceso electoral de un distrito de Lima. A partir de los resultados obtenidos, se mostró la bondad del método propuesto.
El presente trabajo está organizado de la siguiente manera: En el acápite 2 se presenta la revisión de la literatura. En el acápite 3 se describe el método propuesto para la evaluación de la usabilidad del software del voto electrónico presencial, el cual se designó con el nombre de UsabVEP (Método de Evaluación de la Usabilidad del Voto Electrónico Presencial). En el acápite 4 se muestran los resultados de emplear el método UsabVEP. En el acápite 5 se hace una comparación con el método de Nielsen por ser el más representativo en evaluaciones de usabilidad, así como también se propone mejoras de usabilidad al software de voto electrónico. Finalmente en el acápite 6 se exponen algunas conclusiones a las que se llegó.

\section{Revisión de la literatura}

En esta sección se mencionan diferentes estudios de usabilidad y los métodos empleados. Muchos de los estudios son enfocados a distintos ámbitos como por ejemplo: [8], [17] en ámbito de aprendizaje electrónico, [23] en software de salud, [25] en el sector de gobierno electrónico y [2] en el sector bancario; cuyas heurísticas extienden las ya establecidas por Nielsen.

En el trabajo de [5] se evaluaron más de 70 estudios relacionados con heurísticas de usabilidad y se observó que más del 50\% usaron, adaptaron o extendieron las heurísticas propuestas por Nielsen.

Dentro de las pruebas con usuarios finales se tiene el uso de cuestionarios como por ejemplo WAMMI [1], [3] [18], así como [22] quien empleó entrevistas semi-estructuradas y encuestas.

[24] realizó una Evaluación Heurística de Usabilidad del sitio web móvil de bibliotecas de la Universidad de Hong Kong empleando las diez heurísticas de usabilidad desarrollado por Nielsen.

[11] realizó un análisis de la votación del condado de Alabama, se examinó el contenido del sitio web de la elección, la usabilidad, la accesibilidad, y la disponibilidad móvil. Se empleó heurísticas como: Diseño, Navegación, Legibilidad; Idioma; y Facilidad de encontrar en buscadores.

En un estudio realizado por [16], se extendió las heurísticas de Nielsen y tuvo como resultado que el grupo que fue entrenado con las nuevas heurísticas propuestas, encontró un mayor número de problemas de usabilidad para sitios web enfocados al comercio electrónico.

[4] empleó a 24 usuarios los cuales tuvieron que responder un cuestionario de 9 preguntas con puntuación en escala de Likert de 5 puntos a fin de conocer la experiencia del usuario.

Evaluaciones de diseño y usabilidad de una interfaz de múltiples entradas en un entorno idTV como las de [20], consideran nuevas heurísticas de reconocimiento voz, facial, etc. Adicionalmente los usuarios respondie- 
ron un cuestionario de 10 preguntas en escala de Likert de 5 puntos.

Evaluaciones de usabilidad como el de [6] sobre el voto electrónico, emplearon el uso de cuestionario para medir la satisfacción del usuario, así como el uso de tecnologías para experiencia usuario tales como: Audio, Voz, Braile, etc.

Ante la falta de un método de usabilidad enfocado al voto electrónico presencial, el método presenta heurísticas orientadas al problema en estudio. Por lo tanto el presente trabajo de investigación propone un método para la evaluación de la usabilidad del Voto Electrónico Presencial (UsabVEP) el cual permitirá encontrar problemas de usabilidad y de mejorar diferentes aspectos de este tales como: aprendizaje, diseño, accesibilidad, satisfacción del usuario, etc.

\section{Método de evaluación de la Usabilidad del software del Voto Electrónico Presencial}

El enfoque de la investigación es cuantitativo, y el diseño es experimental. En esta sección se describe el método desarrollado para la evaluación de la usabilidad del software del voto electrónico presencial - UsabVEP, y se implementó en el software del proceso electoral realizado en un distrito de Lima. Como se mencionó en la sección de Introducción, autores como [12] y [19] concluyen que los métodos de Evaluación: Heurística y con Prueba de usuarios son las más utilizadas en la valoración de la usabilidad de un software, por ello el método desarrollado incluye las dos estimaciones.

El método UsabVEP presenta dos fases: 1. Evaluación Heurística y 2. Evaluación con Pruebas de usuarios. Para el método se consideran tres actores que participan en el proceso de evaluación, los cuales son:

- Supervisor: Facilita los medios necesarios para la evaluación. A la vez consolida y analiza los resultados de las pruebas, además actúa como moderador en las dos fases del método.

- Evaluador de Heurística: Encargado de identificar problemas de usabilidad en función de las heurísticas elaboradas para el método, así como de calificar los problemas.

- Usuario final: Es el votante que simula una votación electrónica.

\subsection{Fase 1: Evaluación heurística}

El objetivo en esta fase es aplicar heurísticas al software del voto electrónico, con la finalidad de encontrar problemas de usabilidad, que después puedan ser resueltas para mejorar el sistema. Esta fase se desarrolla en ocho actividades, las cuales se muestran en la figura 1 , y que se describen a continuación.

3.1.1. Selección de los expertos (evaluadores): Se requieren entre tres y cinco evaluadores para la evaluación heurística según [14]. El supervisor debe seleccionar a cada evaluador, y éstos deben cumplir la condición de "doble experto", es decir conoce sobre usabilidad y tiene experiencia en votación electrónica.

3.1.2. Capacitación a expertos: Esta actividad consiste en que el supervisor da a conocer a los evaluadores seleccionados las heurísticas de usabilidad del método UsabVEP, las cuales se muestran en la tabla 1. De considerar necesario podrán ańadir nuevas heurísticas, en caso esta no se cumpla esto se convierte en un problema, y se debe especificar el grado de severidad y frecuencia del problema. Según [13], existen tres aspectos para calificar cada problema encontrado, y son los siguientes:

- Severidad del Problema: Representa la gravedad del problema, y puede ser Crítico con valor 4 , Mayor con valor 3, Menor con valor 2, Mínimo con valor 1 y No es un problema con 0 .

- Frecuencia del Problema: Representa el grado de ocurrencia del problema y puede ser: Muy frecuente con valor 4, Frecuente con valor 3, Poco frecuente con valor 2, Inusual con valor $1 \mathrm{e}$ Inexistente con valor 0 .

- Criticidad del Problema: Es la suma de la Severidad más la Frecuencia del Problema.

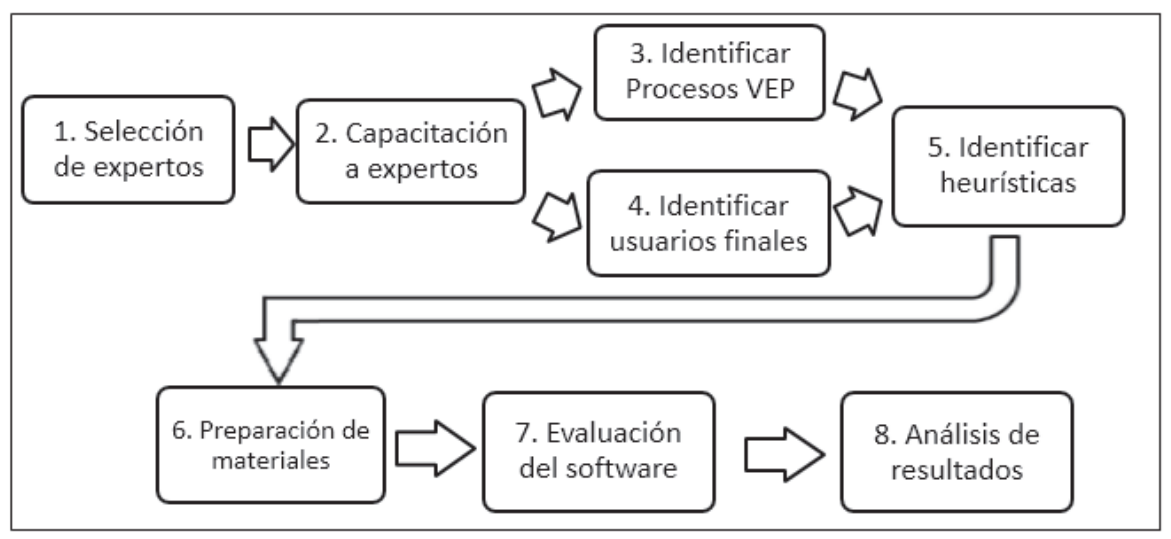

Figura 1. Fase 1 del Método UsabVEP 
3.1.3. Identificación de procesos VEP: Los procesos principales que se presentan en la votación electrónica son: Instalación, sufragio, escrutinio y transmisión. Los evaluadores deben identificar los procesos y tareas que están automatizadas en el sistema del voto electrónico. Por ejemplo, si la identificación del elector que pertenece al proceso de Sufragio se realiza de manera manual, entonces los evaluadores no deben considerar la heurística de Identificación al momento de realizar la evaluación.

\subsubsection{Identificación de usuarios finales: Los} evaluadores deben identificar las características principales de los usuarios finales para determinar qué heurísticas son aplicables al momento de la evaluación. Por ejemplo, en caso de que se implemente el software VEP en una población donde se hable un solo idioma, no se debe considerar la heurística de Idioma o Internacionalización.

\subsubsection{Identificación de heuristicas: Esta acti-} vidad consiste en seleccionar las heurísticas que serán consideradas para la evaluación, elección que se fundamenta en las actividades 3 y 4 . En la tabla 1 se presentan las heurísticas de usabilidad del método UsabVEP que se han elaborado.
Se consideraron algunas heurísticas de uso general, otras se adaptaron de acuerdo a las funcionalidades del sistema, a la organización del proceso, a las facilidades que deben tener los usuarios de diferentes regiones y edades, a la información que debe proporcionar el sistema para la emisión de los resultados, entre otros criterios que se deben tener en cuenta para estos procesos. Los evaluadores pueden agregar otras heurísticas encontradas y considerarlas en la evaluación.

3.1.6. Preparación de materiales: En esta actividad el supervisor proporciona a los evaluadores todo lo necesario para la ejecución de las pruebas, tanto software como hardware y materiales como formularios usados por el método.

\subsubsection{Evaluación del software: Los usuarios} del sistema, es decir los evaluadores, interactúan con el sistema e identifican problemas de usabilidad del software. Se realizan las siguientes tareas: 1) Interacción e identificación de problemas, 2) Consolidación de problemas, 3) Registro de la calificación de problemas y 4) Registro de sugerencias de soluciones.

Tabla 1

Heurísticas UsabVEP

\begin{tabular}{|c|c|c|}
\hline Id & Heurísticas & Descripción \\
\hline $\mathrm{H} 1$ & Diseño de controles & $\begin{array}{l}\text { El diseño debe ser estético y minimalista. El elector debe encontrar fácilmente los elementos en la interfaz. Se } \\
\text { debe visualizar el total de la cédula electoral sin realizar alguna acción. }\end{array}$ \\
\hline $\mathrm{H} 2$ & Diseño del texto & $\begin{array}{l}\text { El texto debe ser legible y claro para el elector, con suficiente contraste entre el fondo y el texto, con adecuados } \\
\text { estilos de texto (tamaño, color, ancho, etc.) }\end{array}$ \\
\hline H3 & Diseño Líquido & $\begin{array}{l}\text { La pantalla debe ajustarse al tamaño horizontal de la pantalla del dispositivo de forma automática, sin una barra } \\
\text { de desplazamiento horizontal. }\end{array}$ \\
\hline $\mathrm{H} 4$ & Diseño de Imágenes & $\begin{array}{l}\text { Las imágenes deben ser de alta resolución para mejor visibilidad, siendo de especial ayuda para electores } \\
\text { iletrados. }\end{array}$ \\
\hline H5 & Visibilidad del estado & $\begin{array}{l}\text { Debe mantener al elector informado de lo que se esté realizando. El usuario no debe de tener dudas de que } \\
\text { está pasando al registrar su voto. }\end{array}$ \\
\hline H6 & Control & $\begin{array}{l}\text { El usuario debe tener libertad al interactuar con el software. Por ejemplo, el elector debe tener la posibilidad de } \\
\text { cambiar su voto antes de su confirmación. }\end{array}$ \\
\hline $\mathrm{H7}$ & Elementos de orientación & $\begin{array}{l}\text { Deben existir elementos que permitan al elector interactuar intuitivamente, saber dónde se encuentra y cómo } \\
\text { volver atrás. En caso de algún flujo inadecuado debe haber flujos alternativos para salir del problema. }\end{array}$ \\
\hline H8 & Instructivo & $\begin{array}{l}\text { Se deben tener instrucciones fáciles de entender. Las instrucciones largas y difíciles de entender incrementan el } \\
\text { tiempo en la votación. }\end{array}$ \\
\hline H9 & $\begin{array}{l}\text { Idioma o } \\
\text { Internacionalización }\end{array}$ & $\begin{array}{l}\text { Tener la posibilidad de mostrar las instrucciones al elector en otros idiomas, en caso sea necesario. Las instruc- } \\
\text { ciones pueden ser también por sonido. }\end{array}$ \\
\hline H10 & Cobertura & $\begin{array}{l}\text { La transmisión de resultados puede realizarse desde cualquier local de votación. Sino, debe tener la posibilidad } \\
\text { de consolidar la información en un dispositivo y llevarlo a otro local donde sí se pueda realizar la transmisión. }\end{array}$ \\
\hline H11 & $\begin{array}{l}\text { Audición } \\
\text { o Sonido }\end{array}$ & $\begin{array}{l}\text { Tener la opción de leer las instrucciones al lector e interactuar con el sistema usando el sonido ya sea con voz } \\
\text { humana o con sintetizador de voz. }\end{array}$ \\
\hline H12 & Limitaciones visuales & $\begin{array}{l}\text { Se debe permitir interactuar con electores con limitaciones visuales (Ceguera, baja visión, daltonismo). Tener } \\
\text { una guía auditiva que leerá las instrucciones. }\end{array}$ \\
\hline H13 & Limitaciones motoras & $\begin{array}{l}\text { El sistema debe permitir interactuar electores con limitaciones motoras. Se podrían seleccionar opciones me- } \\
\text { diante reconocimiento de voz o por detección de movimientos de la cabeza mediante giroscopios. }\end{array}$ \\
\hline H14 & Limitaciones cognitivas & $\begin{array}{l}\text { Debe permitir interactuar electores con limitaciones cognitivas (Problemas de lectura, pérdida de memoria, } \\
\text { dislexia). }\end{array}$ \\
\hline H15 & Identificación & $\begin{array}{l}\text { Debe tener la posibilidad de contar con identificación Biométrica o algún otro mecanismo de identificación en } \\
\text { caso haya impugnación del elector. }\end{array}$ \\
\hline $\mathrm{H} 16$ & Contingencia & $\begin{array}{l}\text { Debe tener opciones de contingencia cuando fallen los dispositivos con los que interactúa el sistema para que } \\
\text { continúe el flujo de votación. }\end{array}$ \\
\hline
\end{tabular}




\begin{abstract}
3.1.8. Análisis de resultados: Permite analizar los resultados de las evaluaciones para que posteriormente permitan tomar decisiones acerca de los problemas encontrados. Para un mejor análisis, se pueden elaborar reportes tales como: Total de problemas por heurísticas, Total de heurísticas incumplidas por procesos, Total de problemas encontrados por procesos, Reporte Estadístico de los problemas y Reporte de problemas por categoría de Severidad. Estos reportes ayudan a identificar qué problemas son los más críticos en base al promedio de su criticidad.
\end{abstract}

\subsection{Fase 2: Evaluación con pruebas de usuarios}

En esta fase se evalúa la usabilidad del software mediante un cuestionario que se ha elaborado para el método y que se aplica a una muestra de usuarios del sistema que realizan su voto electrónico presencial. El cuestionario permite evaluar los siguientes aspectos del sistema: 1) Diseño y organización de la información, 2) Terminología utilizada, 3) Aprendizaje por parte del elector, 4) Eficiencia del sistema, y 5) Satisfacción con el sistema del votante.

En la figura 2 se muestran las actividades que se realizan en esta fase del método UsabVEP, los que se detallan a continuación.

\subsubsection{Selección de Usuarios y Roles: Los usua-} rios del sistema de votación electrónica se seleccionan para participar en las pruebas de usabilidad del software. Ellos representan en cantidad a las características de la población electoral como sexo, edad, grado de instrucción y conocimiento en el uso de la tecnología. Estos usarán el sistema, y luego se les aplicará el cuestionario elaborado para medir la usabilidad del software.

\subsubsection{Orientación al usuario: Se explica al} usuario del sistema el propósito de la evaluación y como debe responder el cuestionario.

\subsubsection{Evaluación del usuario: Esta actividad} tiene dos tareas, que se presentan a continuación.

1) Interacción con el software VEP: El usuario interactúa con el sistema y realiza las tareas básicas para emitir un voto electrónico presencial.
2) Calificación del cuestionario: El usuario del sistema deberá de contestar las preguntas elaboradas en el cuestionario propuesto. El cuestionario consta de 16 preguntas las cuales se calificarán de acuerdo a una escala de Likert de cinco puntos. Estas preguntas están agrupadas en las siguientes categorías: Diseño y organización de la Información, Terminología, Aprendizaje, Eficiencia y Satisfacción del usuario. La calificación de las preguntas del cuestionario UsabVEP de acuerdo a la escala de Likert son: Totalmente en desacuerdo (TDA) con valor 1, En desacuerdo (DES) con valor 2, $\mathrm{Ni}$ de acuerdo ni en desacuerdo (ND) con valor 3, De acuerdo (DA) con valor 4, y Totalmente de acuerdo (TA) con valor 5 . En la tabla 2 se muestra el cuestionario elaborado para la evaluación con usuarios.

\subsubsection{Análisis de los resultados: Basándose en} la información que se obtenga del cuestionario UsabVEP completados por los usuarios del sistema, se confeccionarán reportes para analizar los problemas encontrados de usabilidad en esta Fase de la evaluación del software, para posteriormente tomar las acciones correctivas necesarias. Entre los informes que se obtienen son: Reporte estadístico por pregunta y por cada categoría de preguntas. Los estadísticos considerados son: Máximo, mínimo, promedio y medidas de dispersión como desviación de estándar o coeficiente de variación.

\subsection{Implementación del método}

Para realizar la evaluación de usabilidad con el método UsabVEP, se implementó en el software de un proceso electoral realizado en el distrito Santa María del Mar de Lima, en el año 2013. Debido a que en este año se empezó a usar el voto electrónico solamente en este distrito de Lima. En donde se aplicaron las ocho actividades definidas en la fase 1 y las cuatro actividades de la fase 2.

En la fase 1, Evaluación heurística, se seleccionó a cinco evaluadores que cumplen las características de doble experto, se les capacitó con las heurísticas de usabilidad del UsabVEP (ver tabla 1), y se consideraron los procesos de la votación electrónica presencial: Instalación, sufragio, escrutinio y transmisión. Los usuarios del sistema, en esta fase, son los cinco evaluadores se-

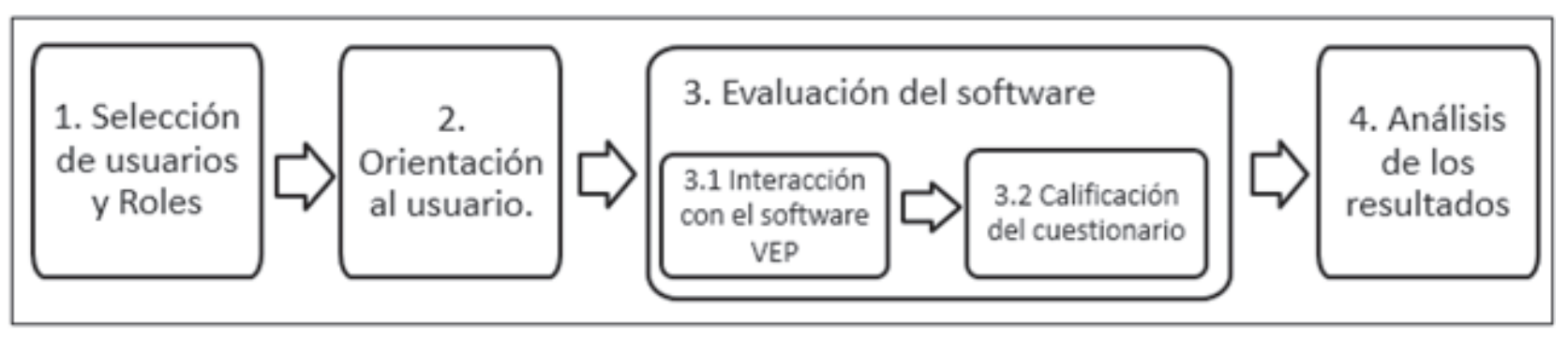

Figura 2. Actividades de la Fase 2 del Método UsabVEP 
leccionados, quienes exploraron las interfaces de cada proceso del sistema, verificaron las heurísticas de la tabla 1 adecuadas al proceso de votación electrónica que se está evaluando, y registraron los problemas encontrados en un formulario de registro de problemas. De esta manera, se realizó la valoración del software en esta fase. En la siguiente sección se presentan los resultados obtenidos y el análisis que se concluye.

En la fase 2, la población electoral en el distrito Santa María del Mar fue de 1422 electores [9], a partir de estos se seleccionó una muestra de treinta usuarios. Estudios como en [7], [8], [19] y [22] emplean entre 12 y 36 usuarios para la evaluación.

A cada usuario seleccionado se le explicó el propósito de la evaluación, luego ellos utilizaron el sistema de votación electrónica simulando la emisión de un voto, y se les solicitó evaluar su experiencia de utilizar el sistema mediante el cuestionario de preguntas relacionadas a la usabilidad del software (ver tabla 2). Para ello se implementaron las cuatro actividades descritas en esta etapa del método UsabVEP, en la siguiente sección se describen los resultados obtenidos en esta fase.

\section{Resultados}

En esta sección se muestran los resultados obtenidos en cada fase de la implementación del método UsabVEP con el software del Voto Electrónico Presencial del Perú en el proceso electoral desarrollado en un distrito de Lima. Esencialmente, se presentan gráficamente los problemas que se han hallado en base a las heurísticas elaboradas para este fin, al mismo tiempo se analizan aquellas faltas u omisiones que deben ser absueltas prioritariamente para una mejor usabilidad del software. A la vez, en esta sección se presenta una comparación del método desarrollado con otro referente en la literatura, y se proponen mejoras al sistema del voto electrónico presencial utilizado.

\subsection{Análisis de resultados de la fase 1}

En la tabla 3, se muestran los problemas encontrados por los evaluadores en esta etapa de la evaluación del software, a la vez se muestran en que proceso del sistema se dieron, y las heurísticas incumplidas. De acuerdo a los problemas obtenidos en la tabla 3 se resumen los resultados, y se resaltan aquellos problemas que se debe priorizar su solución, Se observa que los procesos de Sufragio y Transmisión son los que presentan la mayor cantidad de heurísticas incumplidas y en el proceso de sufragio suceden la mayor cantidad de problemas, que deberían ser tomadas en cuenta para su solución rápida en el software.

El método UsabVEP estableció que los problemas se pueden calificar con los criterios de severidad, frecuencia y criticidad, este último se obtiene de la suma de los dos primeros. Valorando los criterios indicados, se obtiene que los problemas P13, P3, y P11 presentan mayor valor de criticidad, los que deberían ser resueltos con prioridad.

Tabla 2

Cuestionario UsabVEP

\begin{tabular}{|c|c|c|c|c|c|c|}
\hline \multicolumn{2}{|c|}{ Preguntas } & TDA & DES & ND & DA & TA \\
\hline \multicolumn{7}{|c|}{ Diseño y organización de la Información } \\
\hline 1 & La lectura de las instrucciones en pantalla es claramente legible & O & O & ○ & O & $D$ \\
\hline 2 & La secuencia de pantallas es coherente. & ○ & O & ○ & O & 0 \\
\hline 3 & La posición de los mensajes de información y de error en la pantalla es consistente & ○ & ○ & ○ & ○ & O \\
\hline 4 & Fue fácil de encontrar lo que quiero en la pantalla & ○ & O & $\bigcirc$ & $\bigcirc$ & O \\
\hline 5 & El diseño es atractivo & O & $\bigcirc$ & ○ & $\bigcirc$ & O \\
\hline \multicolumn{7}{|c|}{ Terminología } \\
\hline 6 & Los términos empleados en todo el sistema son claramente entendibles & O & O & ○ & O & \\
\hline 7 & La terminología informática está relacionada con la tarea que está haciendo & ○ & ○ & $\bigcirc$ & $\bigcirc$ & ( \\
\hline 8 & El sistema me mantiene informado sobre lo que está haciendo & ○ & $\bigcirc$ & ○ & $\bigcirc$ & O \\
\hline \multicolumn{7}{|c|}{ Aprendizaje } \\
\hline 9 & Creo que el sistema es fácil de aprender para cualquier tipo de persona & $\bigcirc$ & O & $\bigcirc$ & O & O \\
\hline 10 & Fue muy fácil interactuar con el sistema la primera vez que lo use & ○ & O & ○ & $\bigcirc$ & O \\
\hline \multicolumn{7}{|c|}{ Eficiencia } \\
\hline 11 & El sistema responde rápido cuando interactúo & 0 & 0 & O & 0 & O \\
\hline 12 & Este software me ahorró tiempo que si las operaciones lo haría manualmente & O & ○ & $\bigcirc$ & O & O \\
\hline \multicolumn{7}{|c|}{ Satisfacción } \\
\hline 13 & El sistema es muy fácil de usar & $\bigcirc$ & O & O & O & O \\
\hline 14 & Este software de votación electrónica debe reemplazar a la votación manual & ○ & $\bigcirc$ & ○ & $\bigcirc$ & O \\
\hline 15 & Me gustaría usar este sistema la próxima vez & O & O & O & O & $\mathrm{O}$ \\
\hline 16 & Me he sentido seguro de usar este software & O & ○ & O & O & $\mathrm{O}$ \\
\hline
\end{tabular}


Tabla 3

Problemas encontrados en cada proceso, y las heurísticas incumplidas.

\begin{tabular}{|c|c|c|c|c|}
\hline Proceso & $\begin{array}{l}\text { Identificación } \\
\text { de Problema }\end{array}$ & Problema & $\begin{array}{l}\text { Heurísticas } \\
\text { incumplidas }\end{array}$ & Criticidad \\
\hline Instalación & PO & $\begin{array}{l}\text { No se visualiza completamente el nombre de los miembros de mesa cuando } \\
\text { tienen nombres largos. }\end{array}$ & H5 & 3.4 \\
\hline \multirow{10}{*}{ Sufragio } & P1 & No existe la posibilidad de mostrar las instrucciones en otro idioma. & $\mathrm{H9}$ & 2.8 \\
\hline & $\mathrm{P} 2$ & $\begin{array}{l}\text { No se visualiza completamente el nombre de los miembros de mesa cuando } \\
\text { tienen nombres largos. }\end{array}$ & $\mathrm{H} 5$ & 3.5 \\
\hline & P3 & No permite deshacer después de impugnar a un elector por error. & H6 & 5.4 \\
\hline & P4 & $\begin{array}{l}\text { El botón de impugnar quita protagonismo al botón de confirmar la identidad del } \\
\text { elector y parece parte del flujo normal. }\end{array}$ & $\mathrm{H} 2$ & 2.4 \\
\hline & P5 & No permite emitir voto a usuarios con limitaciones visuales. & $\mathrm{H} 12$ & 4.0 \\
\hline & P6 & No permite emitir voto a usuarios con limitaciones motoras. & $\mathrm{H} 13$ & 4.0 \\
\hline & P7 & No permite emitir voto a usuarios con limitaciones cognitivas. & $\mathrm{H} 14$ & 4.0 \\
\hline & P8 & $\begin{array}{l}\text { No permite la identificación biométrica del elector en caso haya impugnación del } \\
\text { elector. }\end{array}$ & $\mathrm{H} 15$ & 3.6 \\
\hline & P9 & $\begin{array}{l}\text { No se indica en qué parte del proceso electoral se encuentra ejecutándose, pero si } \\
\text { indica la actividad que estoy realizando. }\end{array}$ & $\mathrm{H} 5, \mathrm{H} 7$ & 2.0 \\
\hline & P13 & No existe la posibilidad de leer las instrucciones emitiendo sonidos. & $\mathrm{H} 11$ & 7.0 \\
\hline Escrutinio & P10 & $\begin{array}{l}\text { Se encuentra terminología ambigua cuando se están sumando los votos de la } \\
\text { cabina de votación. }\end{array}$ & $\mathrm{H} 8$ & 2.0 \\
\hline \multirow[t]{2}{*}{ Transmisión } & P11 & $\begin{array}{l}\text { La opción de transmitir por Contingencia no realiza una transmisión alterna en } \\
\text { caso la transmisión normal falle. Lo que hace es solo una retransmisión por el } \\
\text { mismo medio, pero si falla este medio no existe contingencia. }\end{array}$ & $\begin{array}{l}\mathrm{H} 1, \mathrm{H} 6, \mathrm{H} 8, \\
\mathrm{H} 16\end{array}$ & 4.6 \\
\hline & P12 & $\begin{array}{l}\text { No existe una visibilidad del progreso de la transmisión ni el porcentaje de trans- } \\
\text { misión, ni cuanto falta por transmitir. }\end{array}$ & $\mathrm{H} 5, \mathrm{H} 7$ & 4.4 \\
\hline
\end{tabular}

En la figura 3 se muestra un gráfico del porcentaje de problemas por categoría de severidad, el que ayuda a visualizar la distribución de la severidad de los problemas que se presentaron en esta evaluación. La categoría "Mayor" representa el mayor porcentaje de problemas de usabilidad del software.

\subsection{Análisis de resultados de la fase 2: Evaluación con usuarios}

De acuerdo con las respuestas obtenidas al aplicar el cuestionario UsabVEP y a su calificación, suministrado a los treinta usuarios que participaron, se elabora el gráfico de estadísticas por preguntas del cuestionario, que se muestra en la figura 4 y que permitirá analizar los problemas de usabilidad obtenidos en esta fase, y para una posterior mejora del software del voto electrónico.
En el método UsabVEP se estableció que a cada pregunta del cuestionario los usuarios del sistema podían calificar con las valoraciones de: TDA con el valor 1 , DES con el valor 2, ND con el valor 3, DA con el valor 4, y TA con el valor 5. En la figura 4 se exhiben los promedios de las calificaciones para cada pregunta realizada por los treinta usuarios seleccionados para que evalúen el software con este método.

Se observa que el promedio de las calificaciones se ubican entre tres y cuatro puntos, así como un máximo de 5 en casi todas las preguntas; por lo que se puede decir que hubo aceptación por parte del usuario. En el gráfico además aparece que los coeficientes de variación de los valores de las preguntas son bajos, lo que significa que hay poca variabilidad entre las calificaciones y los usuarios casi coincidieron en sus respuestas.

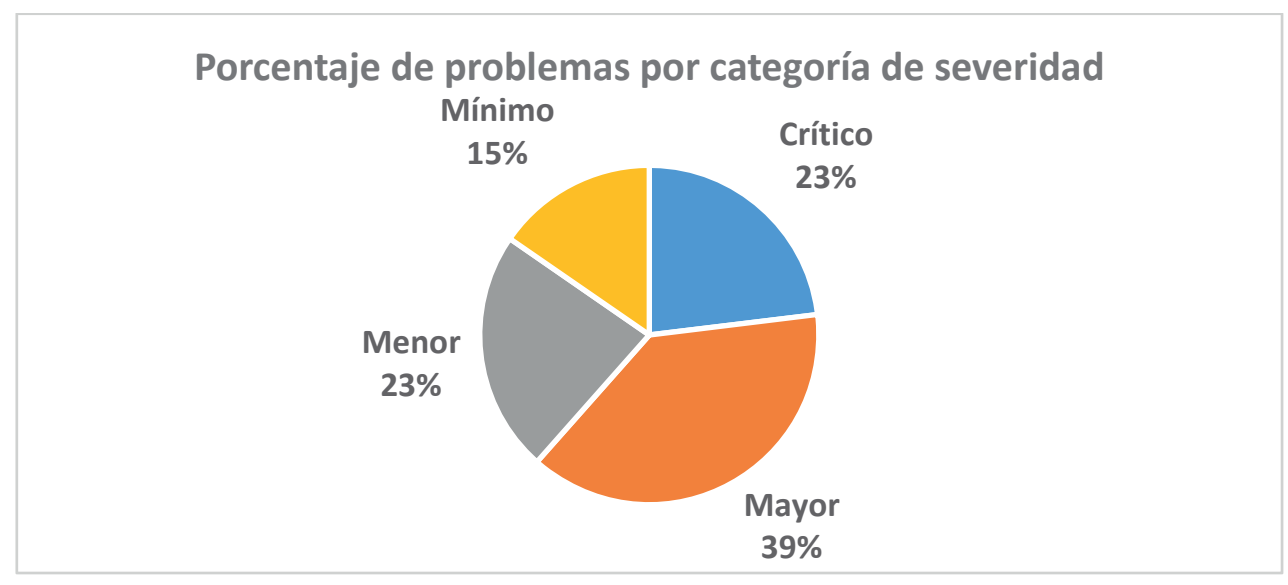

Figura 3. Porcentaje de problemas por categoría de severidad 


\section{Discusión.}

\subsection{Comparación del método}

El método UsabVEP se comparó con el método de Jakob Nielsen [5] [12] por ser el más representativo en evaluaciones de usabilidad, se compararon el total de problemas encontrados aplicando las heurísticas de cada método. El resultado mostrado en la figura 5, se observa que el método propuesto encontró más problemas que el de Nielsen, asimismo se observa que seis problemas fueron hallados solamente con el método UsabVEP, y no pudieron ser hallados mediante el método de Nielsen.

Al comparar ambos métodos se puede afirmar que el método UsabVEP encontró un $46 \%$ más de problemas de usabilidad para el software del voto electrónico presencial. Dichos problemas fueron hallados usando las siguientes heurísticas: Internacionalización, Limitaciones Visuales, Limitaciones motoras, Limitaciones Cognitivas, Identificación, Contingencia y Audición.

\subsection{Propuesta de mejoras al sistema VEP}

En base a los problemas encontrados al aplicar el método UsabVEP al sistema de voto electrónico presen- cial se recomienda realizar mejoras al software evaluado, los que se proponen a continuación:

- Emitir las instrucciones por sonidos para el caso de Quechua hablantes o personas iletradas.

- Tener opciones que emitan sonidos o emplear auriculares u otros artificios de tecnología adaptativa para proveer accesibilidad a usuarios con alguna limitación. Se puede hacer el uso de papeletas electrónicas en Braile. Emplear audífonos para que un elector invidente pueda escuchar su voto y así se mantenga el carácter secreto del voto.

- Tener posibilidad de seleccionar opciones mediante reconocimiento de voz o por detección de movimientos de la cabeza mediante giroscopios para usuarios con limitaciones motoras.

- Se recomienda el uso de tecnología de identificación biométrica como huella dactilar u o reconocimiento de rostros en caso haya impugnación del elector.

- En el proceso de transmisión de resultados, se recomienda usar una barra de progreso indicando el porcentaje de información transferida. En caso no exista comunicación, se debe tener la posibilidad de consolidar la

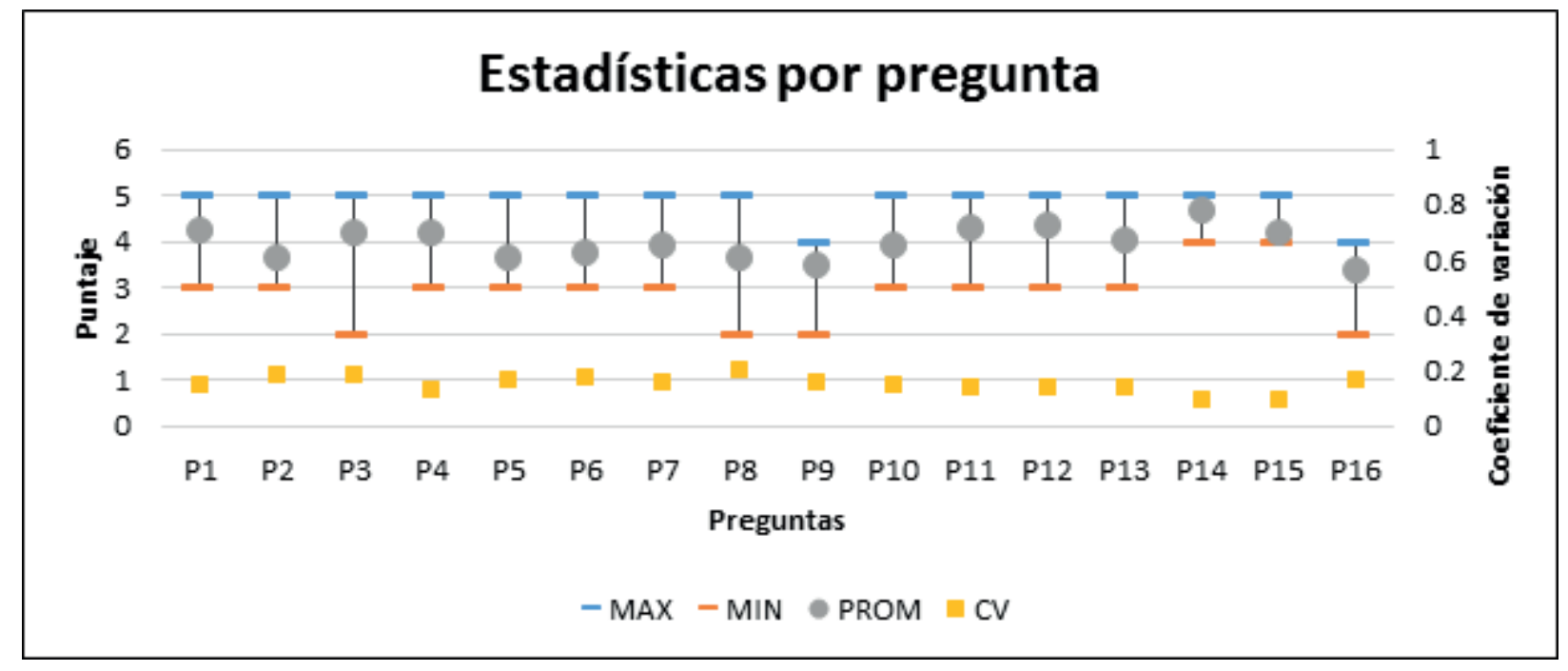

Figura 4. Estadísticas por preguntas del cuestionario

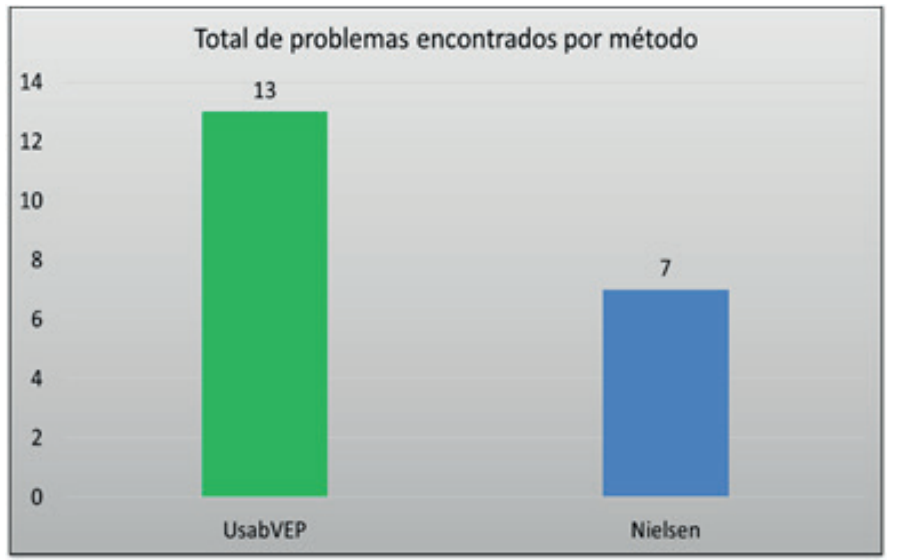

Figura 5. Total de problemas encontrados por método 
información en un dispositivo y llevarlo a otro local en donde sí se pueda realizar la transmisión.

\section{Conclusiones}

Siendo los sistemas de votación electrónica un elemento de apoyo importante en la elección de gobiernos locales, regionales y nacionales. A la vez, la usabilidad mide la calidad en uso de un software. Dos elementos importantes presentes en la realización del trabajo actual.

La contribución consistió en desarrollar un método para evaluar la usabilidad del software del voto electrónico presencial - UsabVEP, con la finalidad de encontrar y analizar problemas de diseño e interactividad al utilizar el sistema, que después puedan ser resueltas para mejorarlo.

La novedad en esta labor se fundamenta en el estudio de métodos de evaluación de la calidad de software de uso general, y se aplicaron a procesos electorales.

El método UsabVEP se implementó en el software de un proceso electoral realizado en un distrito de Lima, para evaluar la usabilidad del sistema se analizaron los resultados, y para este caso se hallaron 13 problemas del software en la fase de evaluación heurística; de los cuales un 23\% fueron críticos, y un 39\% fueron problemas de consideración. En la fase de evaluación con prueba de usuarios, según el análisis de los resultados hubo aceptación mayoritaria del sistema por parte de los usuarios que simulan el voto electrónico presencial.

Basándose en los resultados obtenidos por la aplicación del método al sistema VEP se propusieron mejoras al sistema evaluado, principalmente por los problemas encontrados en la fase de Evaluación heurística.

El método UsabVEP se comparó con el método de Jakob Nielsen por ser el más representativo en evaluaciones de usabilidad, se encontró un $46 \%$ más de problemas de usabilidad para el software del voto electrónico presencial empleando las heurísticas de UsabVEP, que con los obtenidos aplicando las heurísticas de Nielsen.

Se puede concluir que el método UsabVEP contribuye en gran manera con la mejora de un software para el proceso del voto electrónico presencial, superando de esta manera dificultades que podrían encontrar los votantes que interactúan con el sistema como instrucciones ilegibles o difíciles de entender, dificultad de interactuar con usuarios con limitaciones visuales o motoras, dificultad de aprender a usarlo o insatisfacción del usuario final.

Algunas recomendaciones y trabajos futuros que se derivan de la labor realizada en la investigación, se mencionan a continuación.

El método UsabVEP propuso heurísticas que son guías no solo para los procesos electorales peruanos sino que pueden ser adaptados para cualquier país teniendo en cuenta sus leyes. Además, podría ser aplicado para diferentes modalidades del voto electrónico, por ejemplo el voto electrónico no presencial (VENP), el cual los electores pueden realizar la emisión de su voto desde su hogar o usando dispositivos móviles.

Permite considerar nuevas heurísticas que vayan de acuerdo con el desarrollo tecnológico como por ejemplo el reconocimiento de la voz humana para elegir las opciones o dispositivos que se desarrollen en el futuro que impliquen nuevas interacciones hombre-máquina.

Posibilita usar medidas de eficiencia como tasa de errores, tasa de éxito, tiempo en realizar alguna tarea (tiempo de emisión del voto por parte del elector).

\section{Referencias}

[1] Ahmet Mentes, Aykut H. Turan. "Assessing the usability of university websites: An empirical study on Namik Kemal University", TOJET: The Turkish Online Journal of Educational Technology - July 2012, volume 11 Issue 3.

[2] Altin. "Usability guideline for banking software design". Computers in Human Behavior. 2016

[3] Claridge, N. Y Kirakowski, J., 2018. WAMMI: Website Analysis and MeasureMent. [En línea] Consultado el: 02-de marzo de 2018. Disponible en: http://www.wammi.com/samples/ index.html

[4] Daly C., Zapata C., Paz F., 2017. Improving the Usability in a Video Game Through Continuous Usability Evaluations. En: 6th International Conference, DUXU 2017 Held as Part of $\mathrm{HCl}$ International 2017. Vancouver, Canada, p. 387-397.

[5] Hermawati S., Lawson G., 2016. Establishing usability heuristics for heuristics evaluation in a specific domain: Is there a consensus?, Applied Ergonomics, vol 56: p. 34-51.

[6] Hsu J., Bronson G., 2017 E-Voting Technologies Usability: A Critical Element for Enabling Successful Elections. En: Third International Conference on Business Management and Technology. Vancouver, Canada, p. 61-78.

[7] Huang Z., Benyoucef M., 2014. Usability and credibility of e-government websites. Government Information Quarterly, 31(4): p. 584-595.

[8] Hussain M., Abdul M., 2014. Students' Assessment on the Usability of E-learning Websites. Procedia Social and Behavioral Sciences, vol. 141, p. 916 - 922.

[9] INSTITUTO Nacional de Estadística e Informática (INEI), 2013. Compendio estadístico del Perú 2013. [En línea] 2013. Lima: Eds. INEI. Consultado el: 02-de marzo de 2018. Disponible en: https://www.inei.gob.pe/media/MenuRecursivo/ publicaciones_digitales/Est/Lib1097/libro.pdf

[10] ISO International Organization for Standardization. ISO/IEC 25010. [En línea] 2011. Consultado el: 01-de diciembre de 2018. Disponible en: https://www.iso.org/standard/35733. html

[11] A. King, Norman E. Youngblood. "E-government in Alabama: An analysis of county voting and election website content, usability, accessibility, and mobile readiness". Government Information Quarterly (2016).

[12] Nielsen, J., 1995 a. How to Conduct a Heuristic Evaluation. En John Wiley \& Sons. Usability Inspection Methods, New York. [En línea] Consultado el: 02-de marzo de 2018. 
Disponible en: http//www.nngroup.com/articles/how-to-conduct-a-heuristic-evaluation/

[13] Nielsen, J. Severity Ratings., 2012. En John Wiley \& Sons. Usability Inspection Methods, New York, 1995 b.

[14] Nielsen, J. How Many Test Users in a Usability Study?. [En línea] Nielsen Norman Group: Eds. Consultado el: 02 de marzo de 2018. Disponible en: http://www.nngroup.com/articles/ how-many-test-users/

[15] OFICINA Nacional de Procesos Electorales (ONPE), 2017. : Voto Electrónico. [En línea] Consultado el: 02 de marzo de 2018. Disponible en: www.web.onpe.gob.pe/modElecciones/ elecciones/elecciones2017/em-dic2017/voto-electronico.

[16] Paz F.A., Pow-Sang J.A. (2017) Comparing the Effectiveness and Accuracy of New Usability Heuristics. In: Nunes I. (eds) Advances in Human Factors and System Interactions. Advances in Intelligent Systems and Computing, vol 497. Springer, Cham.

[17] Rodríguez G., Perez J., Cueva S., Torres R. A framework for improving web accessibility and usability of Open Course Ware sites. Computers \& Education. Volume 109, June 2017. $197-215$

[18] Sharmistha Roy, Prasant Kumar Pattnaik, Rajib Mall. "A quantitative approach to evaluate usability of academic websites based on human perception", Egyptian Informatics Journal (2014), 159-167

[19] Tan W., Liu D., Bishu R.,2009. Web evaluation: Heuristic evaluation vs. user testing. International Journal of Industrial Ergonomics, 39 (4): p. 621-627.
[20] Téllez J.F., Montoya J.C., Trefftz H. (2017) Design and Usability Evaluation of a Multi-input Interface in an idTV Environment. In: Abásolo M., Almeida P., Pina Amargós J. (eds) Applications and Usability of Interactive TV. jAUTI 2016. Communications in Computer and Information Science, vol 689. Springer, Cham

[21] Vasco Gobierno (Eusko Jaurlaritza), 2018. Voto electrónico en el Mundo. Consultado el 06 de Mayo 2019. Disponible en : http://www.euskadi.eus/informacion/voto-electronico-voto-electronico-en-el-mundo/web01-a2haukon/es/

[22] Walji M., Kalenderian, E., Piotrowski M., Tran D., Kookal K., Tokede O., White J., Vaderhobli R., Ramoni R., Stark P., Kimmes N., Lagerweij M., Patel V., 2014. Are three methods better than one? A comparative assessment of usability evaluation methods in an HER. International Jounal of Medical Informatics, 83(5): p 361-367.

[23] Schnall R, Cho H, Liu J. Health Information Technology Usability Evaluation Scale (Health-ITUES) for Usability Assessment of Mobile Health Technology: Validation Study. Journal of Medical Internet Research. Published on 05.01.18 in Vol 6, No 1 (2018): January

[24] Yin Fung Reese Hoi, Dickson K.W. Chiu, Eddie H.T. Ko, Kevin K.W. Ho, Patrick Lo. "Heuristic Usability Evaluation of University of Hong Kong Libraries' Mobile Website". The Journal of Academic Librarianship. 2016

[25] Zhao Huang, Morad Benyoucef, "Usability and credibility of e-government websites", Government Information Quarterly. (2014) 584-595 